\title{
ASSESSMENT STUDY OF SUPERCONDUCTING
} FAULT-CURRENT LIMITERS OPERATING AT 77K

R.F. Giese

Argonne National Laboratory Argonne, IL 60439
M. Runde

Norwegian Electric Power Research Institute Trondheim, Norway

The possible impact of nitrogen-cooled superconductors on the design and cost of superconducting fault-current limiters is assessed by considering the technical specifications such devices must meet and by comparing material properties of $77-\mathrm{K}$ and $4-\mathrm{K}$ superconductors. The main advantage of operating superconductors at $77 \mathrm{~K}$ is that the refrigeration operating cost is reduced by a factor of up to 25 , and the refrigeration capital cost is reduced by a factor of up to 10 . In addition, the heat capacity at $77 \mathrm{~K}$ is several orders of magnitude larger than at $4 \mathrm{~K}$. This phenomenon increases conductor stability against flux jumps but makes switching from the superconducting to normal state slow and difficult. Consequently, a high critical current density, probably at least $10^{5} \mathrm{~A} / \mathrm{cm}^{2}$. may be required.

As electric power systems grow and become more interconnected, fault currents increase. Prudent precautions for handling these increasing fault currents often require the costly replacement of substation equipment or the imposition of changes in the configuration of the system that lead to decreased operational flexibility and lower reliability. Consequently, the need for a device that can prevent the buildup of fault currents has been obvious for years. A number of fault-current limiter (FCL) concepts have been proposed, several incorporating superconducting materials. Some superconducting FCLs have been 


\section{DISCLAIMER}

This report was prepared as an account of work sponsored by an agency of the United States Government. Neither the United States Government nor any agency thereof, nor any of their employees, makes any warranty, express or implied, or assumes any legal liability or responsibility for the accuracy, completeness, or usefulness of any information, apparatus, product, or process disclosed, or represents that its use would not infringe privately owned rights. Reference herein to any specific commercial product, process, or service by trade name, trademark, manufacturer, or otherwise does not necessarily constitute or imply its endorsement, recomrnendation, or favoring by the United States Government or any agency thereof. The views and opinions of authors expressed herein do not necessarily state or reflect those of the United States Government or any agency thereof. 
built as small-scale models, but most of the designs are purely "paper concepts. Only two full-scale devices have been bullt, both single-phase prototypes with ratings in the lower end of the distribution system range. The discovery in 1987 of superconductors with transition temperatures above the boiling point of nitrogen has stimulated renewed interest in large-scale applications of superconductors, in particular FCLs.

This paper concludes that although thorough cost/benefit analyses of FCLs are lacking, surveys among U.S. utllities indicate that the price that utilities are willing to pay for an FCL is a few times the price of a circuit breaker of corresponding rating. Energy management in a superconducting FCL is a critical. but often ignored, issue. Limiting a fault resistively involves dissipation of large amounts of energy. Although using an air-inductor as the limiting element avoids problems associated with energy dissipation, such devices usually require a resistive, triggering element; energy dissipation within this element will still be a problem. Use of either a resistive or inductive shunt across a resistive superconducting element adds both complexity and cost to a superconducting FCL while providing little, if any, benefit. Thus, the most feasible superconducting FCL concept appears to be an in-line, resistive device. By choosing the resistance to provide a specified temperature rise, one can have a relatively compact design. Finally, high-temperature superconducting wire is presently not available and present knowledge of switching of high-temperature superconductors is incomplete. Thus, superconducting FCLs Lased upon hightemperature superconductors are unlikely to be avallable in the near term. 


\title{
ASSESSMENT STUDY OF SUPERCONDUCTING FAULT-CURRENT LIMITERS OPERATING AT $77 \mathrm{~K}$
}

\author{
R.F. Giese ${ }^{1}$ and M. Runde ${ }^{1,2}$ \\ ${ }^{1}$ Energy Systems Division \\ Argonne National Laboratory \\ Argonne, IL 60439 \\ 2Norwegian Electric Power Research Institute \\ 7034 Trondheim-NTH \\ Norway
}

\begin{abstract}
$\triangle B S T R A C T$
The possible impact of nitrogen-cooled superconductors on the design and cost of superconducting fault-current limiters is assessed by considering the technical specifications such devices must meet and by comparing material properties of $77-\mathrm{K}$ and 4-K superconductors. The main advantage of operating superconductors at $77 \mathrm{~K}$ is that the refrigeration operating cost is reduced by a factor of up to 2.5 , and the refrigeration capital cost is reduced by a factor of up to 10 . The heat capacity at $77 \mathrm{~K}$ is several orders of magnitude larger than at $4 \mathrm{~K}$. This phenomenon increases conductor stability against flux jumps but makes sivitching from the superconducting to normal stcte slow and difficult. Consequently, a high critical current density, probably at least $10^{5} \mathrm{~A} / \mathrm{cm}^{2}$, is required.
\end{abstract}

\section{INTRODUCTION}

As electric power systems grow and become more interconnected, the fault currents (i.e., the currents that would flow if a short circuit occurs) increase. Prudent precautions for handling these increasing fault currents often require the costly replacement of substation equipment or the imposition of changes in the configuration of the system that lead to decreased operational flexibility and lower reliability. Consequently, the need for a device that can prevent the buildup of fault currents has been obvious for years. A number of fault-current limiter (FCL) concepts have been proposed [1], but according to a recent study [2], they all have major cost or perfomnance disadvantages.
Superconducting materials are a part of several of the proposed FCL schemes. Comprehensive lists of publications and patents on superconducting faultcurrent limiters (SCFCLs) have been collected [1], [3]. Some SCFCLs have been built as small-scale models (typically a few amperes at a few hundred volts), but most of the designs are purely "paper concepts" that have not been built or tested. To the authors' knowledge, only two full-scale devices have been built, both single-phase prototypes with ratings in the lower end of the distribution system range [4], [5]. A very comprehensive design study of SCFCLs for high-power transmission systems [3], [6], [7] also clarified several important issues, although no device was buiit.

The discovery in 1987 of superconductors with transition temperatures above the boiling point of nitrogen has stimulated renewed interest in large-scale applications of superconductors. The SCFCL has been mentioned repeatedly as a likely early application of high-temperature superconductors (HTSC) in the power sector. The purpose of this paper is to assess the potential impact of ceramic superconductors on the design and performance of the most relevant SCFCL concepts. The evaluation is performed by (1) comparing material parameters typical for $\mathrm{HTSC}$ at $77 \mathrm{~K}$ with those for low-temperature superconductors (LTSC) at $4 \mathrm{~K}$ and (2) considering how the design and cost of SCFCLs will be affected.

Initially, two example power systems with FCLs are analyzed to establish and clarify the technical specifications that an FCL must meet. Power rating is emphasized because this important parameter has been neglecied in many earlier studies of SCFCLs.

This paper is a summary of a report prepared for the International Energy Agency [8].

\section{TECHNICAL SPECIFICATIONS}

An FCL, whether it employs a superconductor or not, is basically a variable impedance that is installed in series with a circuit breaker in a cubstation. In the case of a fault, its impedance increases to a value at which the fault current is reduced to a level that the breaker can handle. Upon removal of the fault or operation of the circuit breaker, the device should retum to its low-impedance mode within a few power cycles.

Several FCL schemes have been proposed, including resistive, inductive, and combined 
resistive/inductive devices. With respect to the system, a purely resistive FCL is preferable because it makes the circuit less inductive and provides greater limiting of the initial fault-current peak.

A resistive $\mathrm{FCL}$, however, acts as a power load, dissipating large amounts of energy during a fault. Consequently, the duration of the fault-limiting action is a crucial parameter, especially if the device employs superconductors. On the other hand, a purely inductive device does not consume energy, but stores the $60-\mathrm{Hz}$ magnetic energy associated with the fault current. In contrast to the resistive energy, the magnetic energy does not accumulate during successive cycles.

The performance specifications for an $\mathrm{FCL}$ in a power system are most conveniently determined by case studies. Figure 1 and Table I describe the two example systems to be used in the present work. For simplicity, only phase-to-ground faults are considered, and unless otherwise stated, any de offsets are disregarded.

The parameters for the $15-\mathrm{kV}$ distribution system have been determined on the basis of the results of a recent survey of U.S. utilities [1]. The 500-kV transmission system represents an extreme case in terms of having large fault currents. (The $X / R$ ratio is taken from ref. 2.)

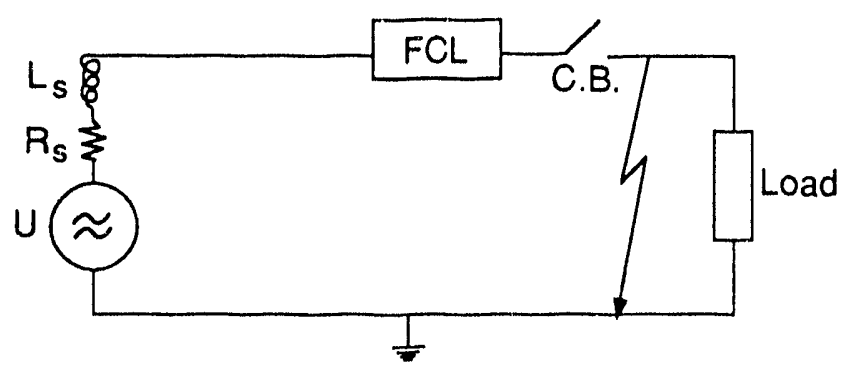

Fig. 1 Circuit Diagram of the Example Systems

TABLE I Parameters for the Distribution and Transmission Example Systems (the power frequency is $60 \mathrm{~Hz}$ )

\begin{tabular}{|c|c|c|}
\hline Parameter & $\begin{array}{l}15-\mathrm{kV} \\
\text { System }\end{array}$ & $\begin{array}{l}500-i \mathrm{~V} \\
\text { System }\end{array}$ \\
\hline Source voltage $\left(k V_{R M S}\right), U$ & 8.7 & 290 \\
\hline $\begin{array}{l}\text { Maximum load current } \\
\left.\text { (A } A_{R M S}\right), I_{n}\end{array}$ & 600 & 3000 \\
\hline Source impedance $(\Omega)$ & 0.43 & 2.4 \\
\hline X/R ratio & 1.7 & 10 \\
\hline Source resistance $(\Omega), R_{S}$ & 0.22 & 0.24 \\
\hline Source inductance $(\mathrm{mH}), \mathrm{L}_{\mathrm{s}}$ & 1.0 & 6.3 \\
\hline Unlimited fault current (KARMS) & 20 & 120 \\
\hline $\begin{array}{l}\text { Maximum limited fault current } \\
\left(\mathrm{KA}_{\mathrm{RMS}}\right)\end{array}$ & 10 & 63 \\
\hline
\end{tabular}

The maximum limited fault currents are set to $10 \mathrm{kA}$ and $63 \mathrm{kA}$ for the distribution and transmission systems, respectively. The highest rating in conventional commercial circuit breakers is $63 \mathrm{kA}$. Table II lists the minimum FCL impedance values for the two example systems.

In a resistive $F C L$, the power dissipated is $\mathrm{RI}^{2}$, where $R$ is the limiting resistance and $I$ is the RMS value of the limited current. The magnetic energy stored in an inductive $\mathrm{FCL}$ is given by $0.5 \mathrm{LI} \mathrm{I}^{2}$, where $\mathrm{L}$ is the limiting inductance and $I$ is the peak value of the limited current. Increasing the impedance of the FCL reduces the limiting current and thereby reduces the energies to be handled by the device. Figure 2 shows the energy dissipated per power cycle in a resistive FCL and the maximum magnetic stored energy in an inductive FCL, both plotted as a function of the limited current. To simplify the calculations, the current ratio between asymmetric and symmetric faults in the inductive device is assumed constant and equal to 1.5 and 1.8 for the $15-\mathrm{kV}$ and the 500-kV systems, respectively.

Figure 2(b) shows that a fault current of $63 \mathrm{kA}$ in the $500-\mathrm{kV}$ system requires a resistive FCL to dissipate $240 \mathrm{MJ}$ per power cycle. (This value is huge; if this energy were dissipated in $1 \mathrm{~m}^{3}$ of water, the temperature would rise by almost $60^{\circ} \mathrm{C}$.) Two power cycles can be considered a minimum time of operation for an FCL, so this device must be able to dissipate $480 \mathrm{MJ}$ per fault. Furthermore, if the relaying system and reclosure procedures are set up in such a way that the FCL in the worst case must operate a second or third time within a few seconds, it must be designed to dissipate energies in the gigajoule range.

By instead applying an inductive device to limit the fault currents to $63 \mathrm{kA}$ in the $500-\mathrm{kV}$ system, up to about $75 \mathrm{MJ}$ of magnetic energy has to be stored in the FCL (see Fig. 2[b]); therefore, a very large device is required. If an iron-core reactor with a maximum energy density of $1 \mathrm{~kJ} / \mathrm{m}^{3}$ in the iron is used, about $75,000 \mathrm{~m}^{3}$ (the size of a two-story warehouse) of iron is required per phase. If the energy is stored in a 1-T field of an air-core reactor, an air volume of $190 \mathrm{~m}^{3}$ is required, and the device is still huge (the size of a large room).

If the fault current in the $500-\mathrm{kV}$ system is limited to $10 \mathrm{kA}$ instead of $63 \mathrm{kA}$, the energies involved are reduced by factors of 5 and 3.5 in the resistive and inductive FCL cases, respectively. However, the energies are still very large.

For the $15-\mathrm{kV}$ FCLs, the energies are typically 250 times lower than those in the $500-\mathrm{kV}$ system (see Fig. 2[a]). Ohmic dissipation of $0.2 \mathrm{MJ}$ to $1 \mathrm{MJ}$ requires some mass and volume, but this is obviously a much simpler task than dealing with gigajoules. Aircore reactors in the range of 1 to $10 \mathrm{mH}$ for $15-\mathrm{kV}$ FCLs are approximately $1 \mathrm{~m}^{3}$ in volume and are commercially available.

Whether or not a utility will install an FCL is largely a question of economics, but information in the literature about acceptable capital and operating costs is 
TAEILE II FCL Impedances (inductances) Required to Reciace the Fault Current to the Predetermined Levels of $10 \mathrm{kA}$ and $63 \mathrm{kA}$

\begin{tabular}{lll}
\hline FCL Type & $\begin{array}{l}15-\mathrm{kV} \\
\text { System }\end{array}$ & $\begin{array}{l}500-\mathrm{kV} \\
\text { System }\end{array}$ \\
\hline $\begin{array}{lll}\text { with res istive } \mathrm{FCL}(\Omega) \\
\text { with inductive } \mathrm{FCL}(\Omega[\mathrm{mH}])\end{array}$ & 0.56 & 3.7 \\
& $0.46(1.2)$ & $2.2(5.8)$
\end{tabular}

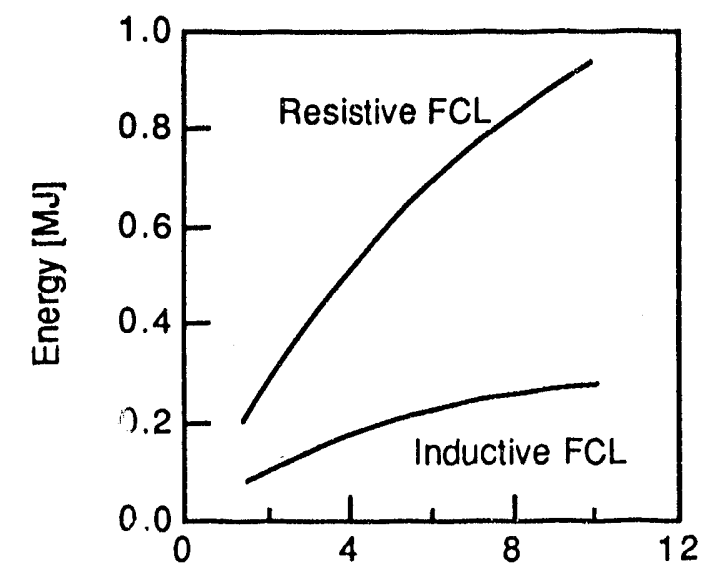

Limited fault current [kA]

(a)

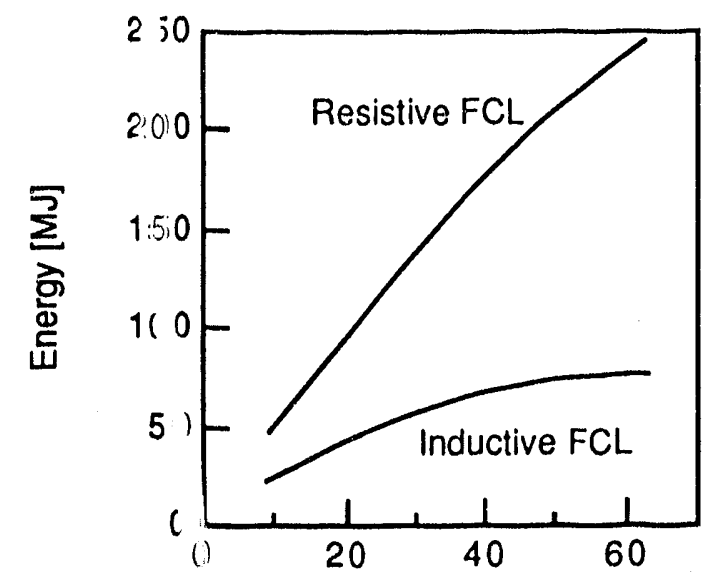

Limited fault current [kA]

(b)

Fig. 2 Energ / Dissipated per Power Cycle in a Resistive FCL and Energy Stored in an Inductive FCL. for the Example Distribution System (a) and Transmission System (b) limited. Two surveys of a number of U.S. utilities [1], [9] indicate that the utilities are not willing to pay more for an FCL than at most a few times the cost of a circuit breaker. The electrical losses during normal operation should not exceed $25 \%$ of the losses in a transformer in the same system [1].

\section{SUPERCONDUCTING FAULT-CURRENT LIMITERS}

Simplified circuit diagrams for four generically different SCFCLs are shown in Fig. 3. In all of these concepts, the superconducting element, displayed as a variable resistance, is inserted directly in series with the power circuit to be protected, and they all make use of a superconducting-to-normal transition. The cryogenic zone is indicated by the broken lines.

Conceptually, the basic resistive SCFCL, as shown in Fig. 3(a), is the simplest design. During normal load, the superconducting element is in its superconducting state and current passes with virtually no losses. In the case of a fault current, the critical current density $\mathrm{J}_{\mathrm{c}}$ of the superconductor is exceeded and the superconductor undergoes a transition to its normal (nonsuperconducting) state, and it is thus transformed to a series resistor that limits the current. A major drawback associated with this concept is that during a fault, all of the energy is dissipated directly into the superconductor. Consequently, the superconductor must have, or be intimately linked to, a large thermal mass, but this arrangement increases the reset time drastically.

The energy dissipated in the superconductor during a fault can be reduced by employing a shunt impedance as the limiting element (see Figs. $3[$ b] $-3[d])$. 'To efficiently commutate the current to the shunt, the normal-state resistance of the superconducting element must be orders of magnitude larger than the shunt. In addition to conventional
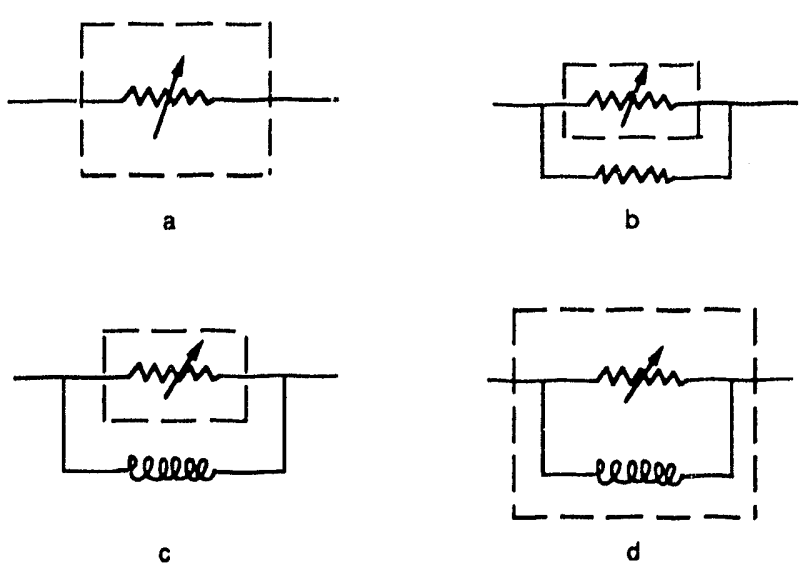

Fig. 3 Different SCFCL Concepts (schematically) 
resistors and air-core reactors, it has been proposed that a superconducting coil be used as a limiting impedance, as shown in Fig. 3(d).

In principle, the SCFCL concepts shown in Fig. 3 can be made self-sensing in that the superconducting-to-normal switching is determined by the critical current density of the superconductor. However, experiments carried out on LTSCs revealed that it may be very difficult to obtain a uniform transition throughout the entire superconducting element [3]. Because of inhomogeneities of various kinds, some small spots always tend to go normal before the bulk. The temperature in these normal-state islands may, because of their much higher resistivity, rise very rapidly, and these hot spots may fuse within microseconds. To ensure a fast, uniform, and complete switching to the normal state during a fault, many SCFCL concepts apply an external trigger. An active trigger causes the critical temperature $T_{c}$, the critical current density $J_{c}$, or the critical magnetic field $\mathrm{H}_{c}$ of the superconductor to be exceeded. Alternatively, the switching can be initiated by applying a magnetic pulse that generates a so-called "flux jump," which eventually quenches the superconductor.

An inherent drawback with the schemes shown in Fig. 3 is that the superconducting element is in-line with the power circuit. Because of this arrangement, there will be ac losses; moreover, heat leaking through the current leads and into the cryostat will cause additional thermal losses.

Several SCFCL concepts in which the superconducting element is not in-line with the power circuit have also been proposed. However, the concepts lead to bulky devices and are in the present study considered infeasible. The saturated iron-core device [4] is more than twice the size of a transformer and is therefore too big and heavy. Inductively linked SCFCLs [10] include a transformer and are disregarded for the same reason. Shielding devices [3], [11] also become prohibitively large because of the vast amounts of iron and/or copper needed.

\section{IMPACT OF HIGH-CRITICAL-TEMPERATURE SUPERCONDUCTORS}

The likely impact of applying the new ceramic superconductors and operating at $77 \mathrm{~K}$ (compared with using conventional low-temperature superconductors at $4 \mathrm{~K}$ ) is discussed in this section. It is assumed that HTSC wires behave according to the "critical state model" proposed by Bean [12] and extensively applied by Wilson [13]. (Data on HTSC materials appear to be consistent with the "critical state model.") The following topics related to the design and operation of SCFCLs are addressed: refrigeration costs, ac losses, stability, switching, and transient temperatures.

\section{Material Properties}

Table III summarizes representative material properties for LTSCs (NbTi) and HTSCs. In the subsequent calculations, $T_{c}$ for these materials is taken
TABLE III Representative Values for Thermal Conductivity k, Heat Capacity c, and Normal-State Resistivity $\rho_{N}$ for Superconductors, Stabilizers, and Substrate Materials at $4 \mathrm{~K}$ and $77 \mathrm{~K}$

\begin{tabular}{llccc} 
Material & $\begin{array}{c}\mathrm{K} \\
(\mathrm{W} / \mathrm{m} \mathrm{K})\end{array}$ & $\begin{array}{c}\mathrm{c} \\
\left(\mathrm{J} / \mathrm{m}^{3} \mathrm{~K}\right)\end{array}$ & $\begin{array}{c}\mathrm{\rho N} \\
\left(10^{-8} \Omega \mathrm{m}\right)\end{array}$ \\
\hline $\mathrm{LTSC}$ & $(4 \mathrm{~K})$ & 0.1 & 5000 & 70 \\
$\mathrm{HTSC}$ & $(77 \mathrm{~K})$ & 2 & $10^{6}$ & 100 \\
$\mathrm{Cu}$ & $(4 \mathrm{~K})$ & 3500 & 400 & 0.038 \\
& $(77 \mathrm{~K})$ & 550 & $1.9 \times 10^{6}$ & 0.25 \\
$\mathrm{Al}_{2} \mathrm{O}_{3}$ & $(4 \mathrm{~K})$ & 220 & 20 & insulating \\
& $(77 \mathrm{~K})$ & 960 & $0.3 \times 10^{6}$ & insulating
\end{tabular}

as $9.5 \mathrm{~K}$ for an LTSC and $92 \mathrm{~K}$ for an HTSC. The HTSC values are obtained from small samples because HTSC wire is not yet available. The values for copper (a commonly used stabilizer) and sapphire (a possible substrate for superconducting films) are also included in Table III.

As shown in Table III, the most evident difference is that the heat capacity at $77 \mathrm{~K}$ is several orders of magnitude higher than the heat capacity at $4 \mathrm{~K}$.

The critical current density $J_{c}$ is also of great importance in the present context. As fabrication processes are optimized, $\mathrm{J}_{\mathrm{c}}$ for HTSCs continues to improve, and nothing indicates that the maximum values have yet been reached. To date, the highest $J_{c}$ values have been obtained from wires drawn from silver tubes filled with HTSC. However, the low resistivity of silver precludes the use of such wires in SCFCLs.

\section{Altemating-Current Losses}

In the concepts shown in Fig. 3, the superconductor passes the full load current under normal operation. Hence, the magnitude of the ac losses (i.e., hysteresis losses and ohmic losses caused by eddy currents if metallic stabilizers are applied) is crucial to the operating costs of the SCFCL.

The electrical losses are primarily determined by the design and operating conditions of the conductor, not by the material properties referred to above. Consequently, in high-quality materials, the losses are expected to be similar for HTSCs and LTSCs.

\section{Refrigeration Costs}

The differences in refrigeration costs depend on (1) the relative thermal loads at $4 \mathrm{~K}$ and $77 \mathrm{~K}$, (2) the relative refrigeration efficiency at $4 \mathrm{~K}$ and $77 \mathrm{~K}$, and (3) the magnitude of refrigeration costs compared with other SCFCL costs.

Thermal Loads: There are three primary sources of thermal load in an SCFCL: (1) thermal conduction through current leads, residual gas, and 
structural components; (2) radiation losses; and (3) internally generated heat (ac losses).

The design of current leads is basically a tradeoff between thermal conduction and ohmic heating. The thermal load P caused by a current lead of crosssectional area $A$ and length 1 is given as follows:

$$
P=\frac{\Delta T \kappa A}{l}+\frac{\rho_{N} I^{2} l}{A}
$$

where $\Delta \mathrm{T}$ is the temperature difference between ambient $(300 \mathrm{~K})$ and the cryogenic zone. Increasing the conductor cross section or reducing its length lowers the ohmic heating and increases the thermal conduction into the cryostat.

If thermal conductivity $\kappa$ and resistivity $\rho_{\mathrm{N}}$ are assumed independent of temperature, the minimum value of $P$ equals

$$
P=2 I \sqrt{\Delta T \rho_{N} K}
$$

for the ratio of cross section to length

$$
\alpha=\frac{A}{I}=I \sqrt{\frac{\rho N}{\Delta T \kappa}} .
$$

By using the parameters for copper in Table III, it appears that the optimal alpha is considerably higher at $77 \mathrm{~K}$, but the thermal loads are the same within the accuracy of this calculation:

$$
\frac{\alpha_{77}}{\alpha_{4}}=8 \text { and } \frac{P_{77}}{P_{4}}=0.9
$$

The thermal load due to thermal conduction through other parts of the cryostat is usually reduced by employing a vacuum shield. When the device is operating at $4 \mathrm{~K}$, all trace gases (except helium) freeze, resulting in a good vacuum. On the other hand, at $77 \mathrm{~K}$, traces of hydrogen, neon, and nitrogen do not freeze. Thus, the thermal conduction losses may be somewhat higher at $77 \mathrm{~K}$ than at $4 \mathrm{~K}$ because of conduction through these residual gases.

When the device is operating at $77 \mathrm{~K}$, the radiation losses between $77 \mathrm{~K}$ and $4 \mathrm{~K}$ are eliminated. The black-body radiation at a temperature $T$ is proportional to $\mathrm{T}^{4}$. Hence, the fraction of the radiation losses that occurs between $77 \mathrm{~K}$ and $4 \mathrm{~K}$ to the radiation losses between room temperature and $77 \mathrm{~K}$ is $\left(77^{4}-4^{4}\right) /\left(300^{4}-77^{4}\right)$ and equals $0.4 \%$. Thus, the reduction in thermal load caused by radiation is marginal.

The thermal load caused by electrical losses is, as discussed above, anticipated to depend mainly on the conductor design and only marginally on whether the device operates at $4 \mathrm{~K}$ or $77 \mathrm{~K}$.
The relative contributions of the various sources to the total thermal load of an SCFCL have been measured and calculated by others. A design study of a 145-kV/2000-A in-line SCFCL [6] estimated the current leads to account for $75 \%$, the cryostat (other thermal conduction losses and radiation losses) for $20 \%$, and the electrical losses for $5 \%$ of the thermal load. Because this device applied a thin superconducting film on an insulating substrate, there were no eddy current losses. In a much smaller prototype device $(25 \mathrm{kV} / 200 \mathrm{~A})$ that used filamentary superconductors in a metallic stabilizer, the corresponding percentages were $12 \%, 82 \%$, and $6 \%$ [5]. Because the current lead losses increase linearly with increasing current, see eq. (2), and the relative contribution of the cryostat losses decreases with its size, the two sets of numbers are in reasonable agreement.

On the basis of these numbers and the above discussion, it can be concluded that with regard to thermal load in an SCFCL, there is no significant difference between operating at $77 \mathrm{~K}$ and operating at 4 $K$. The major sources yield about the same thermal load at the two temperatures. However, as is discussed in the next section, the cost of absorbing the heat is different.

Refrigerator Efficiency: In a survey of cryogenic refrigerators [14], it has been found that their efficiency (relative to the Carnot efficiency) is independent of the lower operating temperature, but efficiency increases with increasing capacity. In this context, capacity refers to the amount of heat that can be absorbed at the lower temperature and rejected at $300 \mathrm{~K}$. Thus, a refrigerator for an SCFCL operating at $77 \mathrm{~K}$ requires 25 times less power input than a refrigerator for an SCFCL operating at $4 \mathrm{~K}$, provided that the thermal loads are the same. The factor 25 is simply the Carnot efficiency between $300 \mathrm{~K}$ and $77 \mathrm{~K}$ relative to $300 \mathrm{~K}$ and $4 \mathrm{~K}$.

The capital costs of the refrigerator have been found to scale approximately as the 0.7 power of the input power [14]. With the same thermal load at $77 \mathrm{~K}$ and $4 \mathrm{~K}$, the refrigerator capital cost is reduced by a factor of 10 .

Refrigeration Costs Relative to Other Costs: The capital cost of the refrigerator relative to the rest of the SCFCL costs decreases with increa'sing complexity of the SCFCL. For a device that employs a high-power shunt, an external triggering unit, overvoltage protection, for example, the refrigerator cost savings at $77 \mathrm{~K}$ relative to $4 \mathrm{~K}$ are unlikely to reduce the total cost of the SCFCL by more than $20 \%$. This percentage is based on the cost estimate for a transmission system SCFCL [3]. If it is possible to make a very simple device, the savings become greater, possibly as high as $50 \%$ for a distribution system SCFCL. However, because little has been published on the cost of SCFCLs, these percentages must only be considered rough estimates.

The cost of refrigeration is expected to dominate the operating cost of an SCFCL. Thus, the savings in SCFCL operating costs by operating at $77 \mathrm{~K}$ are 
expected to be almost as large as the improvement in Camot efficiency.

\section{Stability and Switching}

The superconducting element of the SCFCL concepts shown in Fig. 3 is subjected to stability requirements that, in some senses, are contradictory. On one hand, the superconductor should be stable and remain in its superconducting state during normal load. On the other hand, if a fault occurs, it should switch very rapidly to its normal (nonsuperconducting) state.

Adiabatic Stability: Wires of superconducting materials are inherently unstable unless they have a small cross section. Slight disturbances of almost any kind (thermal, electrical, magnetic, mechanical, etc.) will cause a current-carrying superconductor to quench. A common measure of superconductor stability is the "adiabatic stability criterion." The maximum conductor radius $a_{a d}$ for which a superconducting wire will not exhibit thermal runaway when passing a current density just below $\mathrm{J}_{\mathrm{c}}$ is according to this criterion [13,pg. 141]

$$
a_{a d}=\sqrt{\frac{c\left(T_{c}-T_{o p}\right)}{\mu_{0} J_{c}^{2}}}
$$

where $T_{o p}$ is the operating temperature ( $4 \mathrm{~K}$ or $77 \mathrm{~K}$ ) and $\mu_{0}$ is the permeability of free space. Figure 4 shows $\mathrm{a}_{\mathrm{ad}}$ as a function of $\mathrm{J}_{\mathrm{c}}$ for an LTSC and an HTSC with the parameter values given in Table III.

It appears that HTSC wires are adiabatically stable for diameters 25 times larger than those for LTSC wires.

Switching: The required switching time for the superconductor element in an SCFCL depends on the design, but it typically ranges from a few microseconds to a few milliseconds. If the switching is too fast,

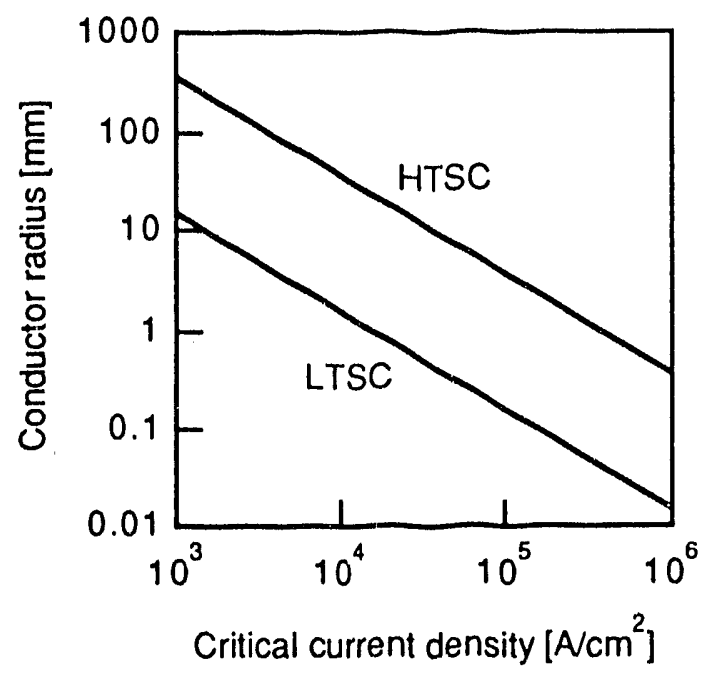

Fig. 4 Maximum Adiabatically Stable Conductor Radius for LTSC at $4 \mathrm{~K}$ and $\mathrm{HTSC}$ at $77 \mathrm{~K}$ overvoltages occur across the device, while the fault currents can build up to unacceptable levels if the switching is too slow.

For both passively and actively triggered switching, the superconductor first enters into the socalled "lossy state." In this state, the superconductor is unstable, and the interaction betweer the current and the magnetic field produces heat, which causes the temperature in the superconductor to rise. However, the superconductor does not reach the full normal-state resistivity $\rho_{N}$ (and, consequently, the full limiting resistance) until $T_{c}$ is exceeded. Thus, in the adiabatic case, the switching time $\tau$ is given as

$$
\tau=\int_{T_{o p}}^{T_{c}} \frac{c}{\rho J^{2}} d T
$$

where $J$ is the current density and $\rho$ is the superconductor resistivity in the lossy state. An orderof-magnitude estimate of $\tau$ can be found by ignoring all temperature dependencies, setting $\rho$ equal to $\rho_{N}$, and setting $\mathrm{J}$ equal to $\mathrm{J}_{\mathrm{c}}$. Figure 5 shows the results of this calculation for a range of $J_{c}$ by using the parameter values in Table III.

Apparently, switching from the superconducting to the normal state is much slower for HTSCs than for LTSCs; moreover, $\tau$ decreases rapidly with increasing $\mathrm{J}_{\mathrm{c}}$.

However, experience with LTSCs has shown that unless active triggering is employed, a superconductor initially goes normal only at one or a few small, weak regions. These nonsuperconducting islands then expand throughout the entire superconductor. The velocity at which the front of the

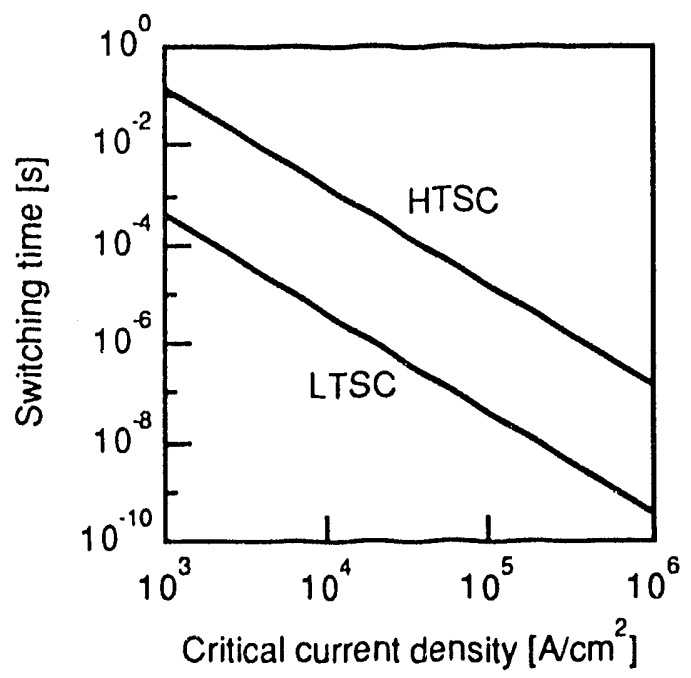

Fig. 5 Switching Times (simplified estimates) for an LTSC and an HTSC 
normal region propagates through the superconductor is called the quench propagation velocity $\mathrm{v}_{\mathrm{q}}$ and has been estimated to equal [13, pg. 206]

$$
v_{q}=\frac{J}{c} \sqrt{\frac{2 \rho_{N} \kappa}{T_{c}-T_{o p}}} .
$$

This relationship is displayed in Fig. 6 for an LTSC and an HTSC.

Hence, thermal processes are expected to proceed much slower in HTSCs than in LTSCs, primarily because of the much greater heat capacities of materials at $77 \mathrm{~K}$ relative to $4 \mathrm{~K}$. As shown above, $\tau$ is two and a half orders of magnitude greater and $v_{g}$ one and a half orders of magnitude smaller at $77 \mathrm{~K}$. These conditions will make the transition of an SCFCL from the low-impedance to the high-impedance mode substantially more difficult with an HTSC than with an LTSC. This is the case both for passive and active triggering. However, as can be seen from Figs. 5 and 6 , both $\tau$ and $v_{q}$ improve radically when $J_{c}$ becomes large. Thus, to obtain sufficiently fast and uniform switching with an HTSC element, a high critical current density is required, probably at least $10^{5} \mathrm{~A} / \mathrm{cm}^{2}$.

\section{Temperature Rise during a Fault}

When the superconductor eventually has been switched to the normal state, the temperature will continue to rise as long as current is flowing. The rate at which the temperature increases is, to the first approximation, proportional to the reciprocal of the heat capacity. Thus, HTSCs are superior to LTSCs because of their much greater heat capacity. However, this assertion is only true if the temperature rise during a fault is small. As will be shown in the next section,

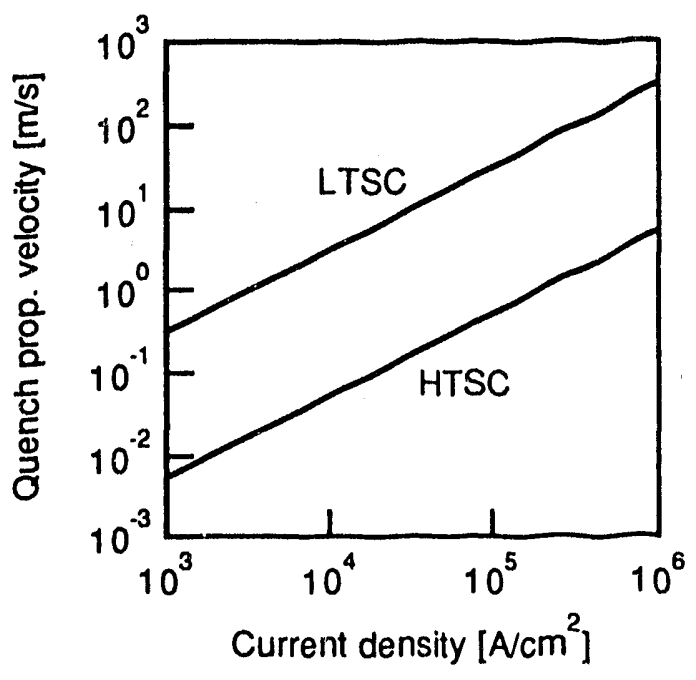

Fig. 6 Quench Propagation Velocities in an LTSC and an HTSC temperature transients of hundreds of kelvin must in some schemes be accepted, even for HTSCs. With such large energy dissipations, whether the coolant is at $4 \mathrm{~K}$ or $77 \mathrm{~K}$ is of minor importance.

\section{DESIGN OF HIGH-CRITICAL-TEMPERATURE FAULT-CURRENT LIMITERS}

Several important design trade-offs that are closely related to the power rating of an SCFCL during a fault will now be discussed; numerical examples will provide the basis for the discussion. The electrical parameters of the example distribution and transmission systems described above are applied, and the discussion is limited to HTSCs and the SCFCL concepts presented in Fig. 3.

\section{Resistive SCFCL}

The basic resistive design in Fig. 3(a) is considered first. The cross-sectional area $\mathrm{A}$ of the superconductor is determined by

$$
I_{c}=J_{c} A
$$

where $I_{c}$ is the highest current the SCFCL should pass without switching to the high-impedance mode. The normal-state resistance $\mathrm{R}$ of a superconductor of length 1 is

$$
\mathrm{R}=\frac{\rho_{\mathrm{N}} \mathrm{l}}{\mathrm{A}}=\frac{\rho_{\mathrm{N}} \mathrm{IJ}_{\mathrm{c}}}{\mathrm{I}_{\mathrm{c}}}
$$

which can be recasted to

$$
l=\frac{I_{c} R}{J_{c} \rho N}
$$

The volume $\mathrm{V}$ of the superconductor can thus be expressed as

$$
\mathrm{V}=\frac{\mathrm{I}_{\mathrm{c}}^{2} \mathrm{R}}{\mathrm{J}_{\mathrm{c}}^{2} \rho_{\mathrm{N}}} .
$$

By assuming a relatively high $\mathrm{J}_{c}$, the FCL resistance specifications given in Table II are easily met for reasonable HTSC wire cross sections and lengths. However, if the power dissipation during a fault is also taken into consideration, major problems are encountered. The temperature rise $\Delta \mathrm{T}$ in time $\Delta \mathrm{t}$ in the superconductor during limiting action is

$$
\frac{\Delta \mathrm{T}}{\Delta \mathrm{t}}=\frac{\mathrm{RI}_{\mathrm{lim}}^{2}}{\mathrm{cV}}
$$

where $\mathrm{I}_{\mathrm{lim}}$ is the limited fault current given by 


$$
I_{\lim }=\frac{U}{\sqrt{\left(R_{s}+R\right)^{2}+\left(\omega L_{s}\right)^{2}}}
$$

where $\omega$ is the angular power frequency. Figure 7 shows the temperature rise in kelvin per millisecond during a fault for the two example systems with the SCFCL resistances given in Table II and for $I_{c}=5 I_{n}$. Adiabatic conditions are assumed, and all material parameters are considered constant and independent of temperature.

As expected, low $\mathrm{J}_{\mathrm{c}}$ values result in a slower heating of the superconductor because its mass is larger. However, because a high current density is required to accomplish sufficiently fast switching, Fig. 7 shows that the Fig. 3(a) design is not technically feasible, if the SCFCLs have the minimum required resistances given in Table II. Even with a current density as low as $10^{4} \mathrm{~A} / \mathrm{cm}^{2}$, the power dissipation will cause the superconductor to evaporate within the first power cycle (17 ms).

The only way to reduce the dissipation and thereby limit the temperature rise in the superconductor is to limit the current even further by increasing the SCFCL resistance. Because $\rho_{N}$ of HTSC cannot be increased without reducing $\mathrm{J}_{\mathrm{c}}$ drastically, this reduction can only be achieved by increasing the length of the superconductor. Figure 8 shows the rate of temperature rise during a fault as a function of the limiting resistance for two values of $J_{c}$. The lower resistance values are those required to limit the current to $10 \mathrm{kA}$ and $63 \mathrm{kA}$, respectively (see Table II).

Limiting the fault current to the nominal load current $I_{n}$ corresponds to SCFCL resistances of about

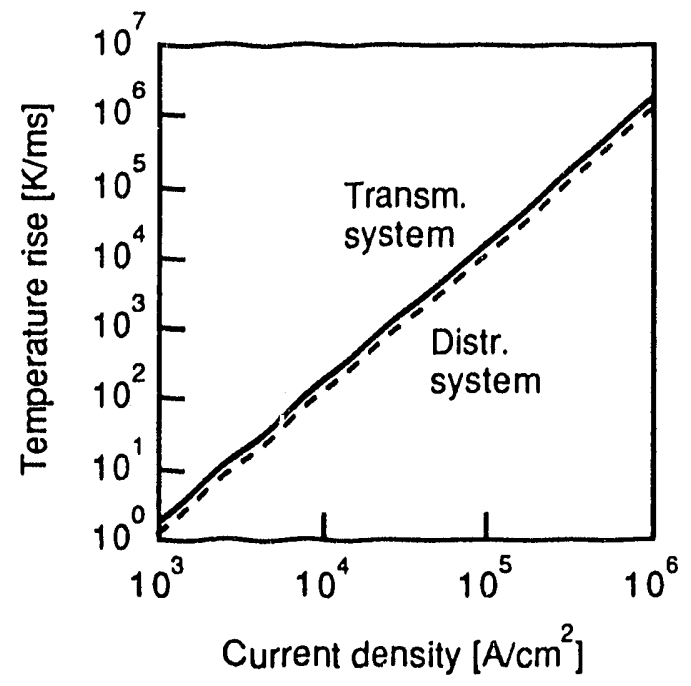

Fig. 7 Temperature Rise in the HTSC Element of the Concept Shown in

Fig. 3(a). (The risistance values are

$0.56 \Omega$ and $2.38 \Omega$ for the distribution and transmission system SCFCL, respectively.)
$14 \Omega$ and $100 \Omega$ for the two systems. The associated temperature rises after two cycles $(33 \mathrm{~ms})$ are, as shown in Fig. 8, still of the order of $10^{3} \mathrm{~K}$, if $\mathrm{J}_{\mathrm{c}}$ is $10^{5}$ $\mathrm{A} / \mathrm{cm}^{2}$. Hence, the current flowing through the superconducting element while in its normal state must be limited to a value significantly smaller than $I_{n}$ to prevent the temperature transients from damaging the superconductor. However, the required superconductor length and volume increase correspondingly. For example, to obtain a limiting resistance of $1 \mathrm{k} \Omega$ in the two systems, the superconductors must be $3 \mathrm{~km}$ and $15 \mathrm{~km}$ long, respectively, assuming $\mathrm{J}_{\mathrm{c}}$ is $10^{5} \mathrm{~A} / \mathrm{cm}^{2}$.

An altemative approach for reducing the amount of current flowing through the superconductor element during a fault is to employ a shunt resistor as the limiting element (see Fig. 3[b]). However, this scheme also requires the superconducting element to have a very high resistance (comparable with the previous design) to ensure that most of the current is commutated to the shunt. The shunt resistance must at least be as specified in Table II.

Although the superconductor in both the shunted and the unshunted resistive SCFCL must perform a rather extreme current limiting, it will still be very difficult, if not impossible, to obtain reset times that are less than several seconds.

Furthermore, in a compact SCFCL design, the substantial wire lengths necessary to obtain such a high resistance of the superconducting element are inevitably associated with a certain stray inductance. In the shunted device of Fig. 3(b), the stray inductance may cause serious problems in the early stages of a fault. As explained in an earlier study [3], the stray inductance of the superconducting element largely determines how rapidly the fault current commutates to the shunt

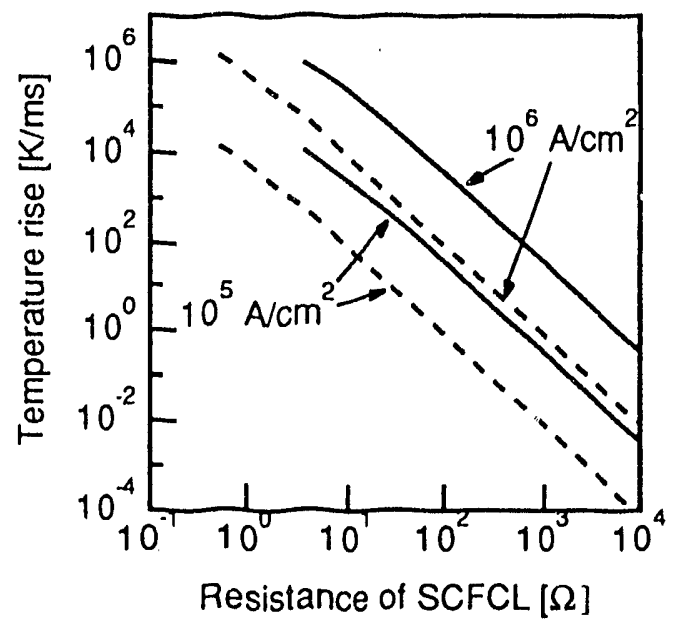

Fig. 8 Temperature Rise per Millisecond in the HTSC Element of the Concept Shown in Fig. 3(a) as a Function of the Limiting Resistance for the Example Distribution System (broken lines) and Transmission System (solid lines) 
resistor. An inductance that is too large may cause the superconductor to fuse before the current is effectively transferred to the high-power shunt.

\section{Inductive SCFCL}

By applying an inductive shunt instead of a resistive SCFCL (see Fig. 3[c] and 3[d]), the L/R ratio increases. (Here, $\mathrm{L}$ includes both the stray inductance in the resistive SCFCL and the limiting inductance in the inductive SCFCL.) Thus, the current commutation to the shunt during a fault becomes much slower when employing an inductive shunt. Consequently, the problems related to energy dissipation in the superconducting element in the early stages of a fault are even greater in this design.

Since the SCFCL already has a cryostat, an interesting scheme is to use an air-core reactor with superconducting windings, as shown in Fig 3(d). The reactor remains in its superconducting state during a fault; the current is limited by its impedance. Compared with applying ambient temperature air-core reactors (Fig. 3[c]), this procedure may lead to a more compact SCFCL design because the ampere-turns and the magnetic energy density can be increased substantially. Doubling the magnetic flux density reduces the required reactor volume by a factor of four, but at the expense of higher structural requirements for balancing the increased magnetic forces between the conductors. The engineering problems related to construction of large high-field (several tesla) superconducting coils are significant and increase rapidly with the size of the coil. The combined volume and flux density for the transmission system SCFCL discussed here is clearly beyond present conventional technology.

Most of the potential difficulties related to the switching and resetting of the superconducting element exist also in the inductive schemes of Fig. 3, because the inductive SCFCLs rely on the same superconducting-to-normal transition. Although these devices limit the fault current inductively, the problems associated with the power dissipation in resistive SCFCLs generally remain.

\section{Cost Considerations and Competing Technology}

The superconducting element in the concepts shown in Fig. 3 (b)-(d) is essentially only a switch that commutates the fault current to the limiting impedance. Thus, the large energies associated with the limiting action are handled by the shunt, which causes the shunt to become large and expensive. In a study of a transmission system SCFCL [3], the shunt resistor was found to account for about half the total SCFCL cost.

Consequently, the capital cost of the SCFCL can be reduced significantly if the shunt impedance can be avoided. To make the shuntless concept of Fig. 3(a) technically feasible, the fault current must be limited to a small fraction of the nominal load current, and the reset time will probably be at least on the order of seconds. For some applications, as for example in the protection of underground transmission cables, long reset times may, however, be fully acceptable.

Other factors with great impact on the capital cost of an SCFCL include the need for an external trigger and the need to install capacitors across the device to reduce overvoltages.

Competing technology to the SCFCL exists, at least at the distribution system level. The feasibility of using gate tum-off thyristors to commutate the fault current to a limiting shunt has recently been demonstrated [1], [15]. Furthermore, a novel and inexpensive arc runner scheme for fault-current limiting has been tested [16]. These and other concepts create strict cost and reliability requirements that an SCFCL must meet to be competitive. For example, the thyristors that essentially have the same function as the superconductor cost around $\$ 40,000$ (U.S.) [1] for a three-phase FCL rated for the example distribution system. Furthermore, the losses in this device are about $0.05 \%$ of the throughput power. Consequently, the refrigeration costs of an SCFCL should not exceed this value.

The extent to which these FCL schemes can be scaled up to high-power transmission systems is unclear.

\section{CONCLUSIONS}

The following conclusions were drawn on the basis of this work:

- Thorough cost/benefit analyses of FCLs are lacking, but surveys among U.S. utilities indicate that the price the utilities are willing to pay for an FCL is at most a few times the price of a circuit breaker of corresponding rating.

- The energy management in an SCFCL is a critical but often ignored issue.

- Although using an air-inductor (coil) as the limiting element avoids problems associated with energy dissipation during faults, such devices usually require a resistive, triggering element; energy dissipation within this element will still be a problem.

- Use of either a resistive or inductive shunt across a resistive superconducting element adds complexity and cost to an SCFCL while providing little, if any, benefit.

- The most feasible SCFCL concepi appears to be an in-line, resistive device. By choosing the resistance to provide a specified temperature rise, one can have a relatively compact ciesign.

- The refrigerator operating cost may be reduced by a factor of up to 25 and the refrigerator capital cost by a factor of up to 10 for an SCFCL operating at $77 \mathrm{~K}$ compared with a device operating at $4 \mathrm{~K}$. 
- The several orders of magnitude greater heat capacity of HTSCs, compared with LTSCs, improves the stability of the superconductor but makes switching to the normal state slow and difficult.

- A high critical current density, probably at least $10^{5} \mathrm{~A} / \mathrm{cm}^{2}$, will likely be required to achieve adequate switching speed and an acceptable superconductor volume.

- HTSC wire is not yet available, and the present knowledge of switching of HTSCs is incomplete. Therefore, SCFCLs using HTSCs are unlikely to be available in the near term.

\section{ACKNOWLEDGMENTS}

The authors wish to thank E.P. Dick for fruitful comments and discussions. The results presented in this paper are based upon work done under the International Energy Agency's (IEA's) Implementing Agreement for a Cooperative Programme for Assessing the Impacts of High-Temperature Superconductivity on the Electric Power Sector. This work was supported, in part, by the U.S. Department of Energy, Assistant Secretary for Conservation and Renewable Energy, under contract W-31-109-Eng-38, as well as by the following participating countries: Canada, Denmark, Finland, Germany, Italy, Japan, the Netherlands, Norway, Sweden, Switzerland, Turkey, and the United Kingdom. One of the authors (M.R.) gratefully acknowledges partial support from the Royal Norwegian Council for Scientific and Industrial Resiparch.

\section{REFERENCES}

[1] Study of Fault-Current-Limiting Techniques, EPRI EL-6903, 1990.

[2] Fault Current Limiters - Basic Concepts and Associated Technologies, EPRI EL-6275, 1989.

[3] Superconducting Fault-Current Limiter, DOE/ET/29063-1, 1982.

[4] B.P. Raju, K.C. Parton, and T.C. Bartram, "A Current Limiting Device Using Superconducting D.C. Bias - Applications and Prospects," Paper no. 82 WM 098-2, IEEE PES Winter Meeting. 1982.

[5] E. Thuries, V.D. Pham, Y. Laumond, T. Verhaege, A. Fevrier, M. Collet, and $M$. Bekhaled, "Towards the Superconducting Fault Current Limiter," IEEE Transactions on Power Delivery, vol. 6, pp. 801-808, 1991.

[6] Superconducting Fault Current Limiter, EPRI EL-329, 1976.

[7] K.E. Gray and D.E. Fowler, "A Superconducting Fault-Current Limiter," J. Appl. Phys., yol. 49, pp. 2546-2550, 1978.
[8] R.F, Giese and M. Runde, Fault-Current Limiters, prepared for the participating signatories of the International Energy Agency by Argonne National Laboratory, Jan. 1992.

[9] Effects of Reduced Fault Duration Upon PowerSystem Components, EPRI EL-2772, 1982.

[10] G.C. Damstra, A. Greenwood, A. Sabot, and H.-H. Schramm, "Superconducting Technology for Current Limiters and Switchgear," CIGRE, 1990.

[11] Y.A. Bashkirov, L.S. Fleishman, T.Y. Patsayeva, A.N. Soblolev, and A.B. Vdovin, "Current-Limiting Reactor Based on High- $\mathrm{T}_{c}$ Superconducters," IEEE Transactions on Magnetics, vol. 27, pp. 1089-1092, 1991.

[12] C.P. Bean, "Magnetization of Hard Superconductors," Phys. Rev. Lett., vol. 8, pp. 250253, 1962.

[13] M.N. Wilson, Superconducting Magnets, Oxford, U.K.: Claredon Press, 1983.

[1:i] T.R. Strobridge, Cryogenic Refrigerators - An Updated Study, NBS Technical Note, 1976.

[15] P.G. Slade, J.L. Wu, E.J. Stacey, W.F. Stubler, R.E. Voshall, J.J. Bonk, J. Porter, and L. Hong, "The Utility Requirements for a distribution Fault Current Limiter," IEEE Transactions on Power Delivery (in press).

[16] N. Engelman, E. Schreurs, and B. Drugge, "Field Test Results for a Multi-shot $12.47 \mathrm{kV}$ Fault Current Limiter," IEEE Transactions on Power Delivery, vol. 6, pp. 1081-1087, 1991.

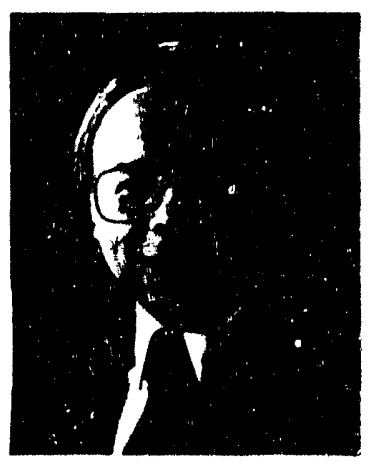

Robert F. Giese was bom in 1943. Ile was graduated from the University of Wisconsin with a degree in Applied Mathematics and Engineering Physics in 1965 . He earned his Ph.D. at Stanford University in 1974. Currently, Dr. Giese is a Systems Analyst in the Energy Systems Division of Argonne National Laboratory.

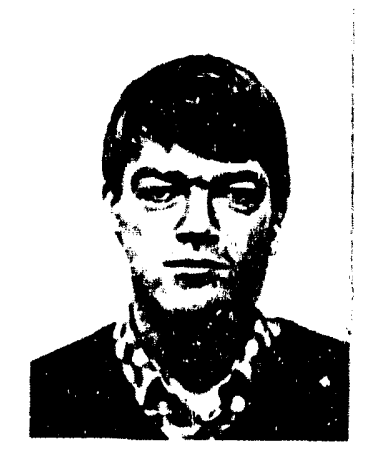

Magne Runde was bom in 1958. He received the M.S. and Dr. Ing. degrees from the Norwegian Institute of Technology in 1984 and 1987. respectively. He is presently with the Norwegian Electric Power Research Institute in Trondheim, Norway. 

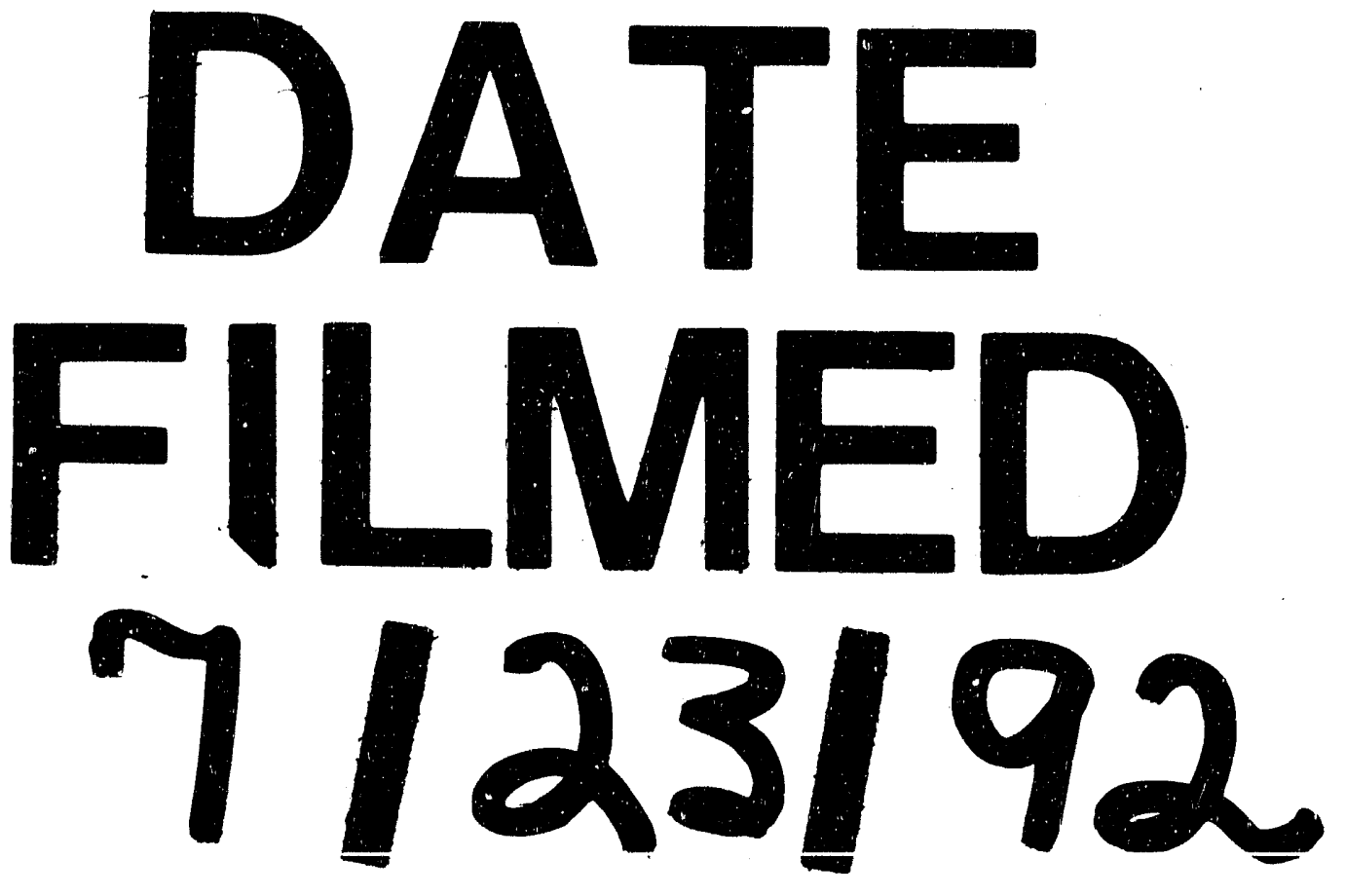
